\title{
Correction to: Methanolic extracts of a selected Egyptian Vicia faba cultivar mitigate the oxidative/inflammatory burden and afford neuroprotection in a mouse model of Parkinson's disease
}

\author{
Essam Abdel-Sattar ${ }^{1,6}$ (D) Engy A. Mahrous ${ }^{1,6} \cdot$ Mareena M. Thabet $^{2}$ - Dina M. Yousry Elnaggar ${ }^{2,9}$. \\ Amal M. Youssef ${ }^{3} \cdot$ Reda Elhawary $^{4}$ Sawsan A. Zaitone ${ }^{5,6} \cdot$ Celia Rodríguez-Pérez $^{7,8} \cdot$ Antonio Segura-Carretero $^{7,8}$. \\ Reham Hassan Mekky ${ }^{2,7}$
}

Published online: 6 March 2022

(c) Springer Nature Switzerland AG 2022

\section{Correction to: Inflammopharmacology (2021) 29:221-235 https://doi.org/10.1007/s10787-020-00768-6}

Addition of an affiliation to an author:

The author Dina M. Yousry Elnaggar should read "Dina M. Yousry Elnaggar ${ }^{2,9 "}$.

${ }^{9}$ Department of Pharmacognosy, Faculty of Pharmacy, Al-Azhar University, Cairo, Egypt.

The author Dina Elnaggar originally belongs to the AlAzhar University. While submitting the original paper the author was working at the Russian University on a loan stint basis. The author did not realize at the time of publication that both universities should be mentioned and this only came to attention while revising the portfolio for the promotion process. The author apologizes for any inconvenience caused.

The original article has been corrected.

Publisher's Note Springer Nature remains neutral with regard to jurisdictional claims in published maps and institutional affiliations.
The original article can be found online at https://doi.org/10.1007/ s10787-020-00768-6.

Essam Abdel-Sattar

essam.abdelsattar@pharma.cu.edu.eg

1 Department of Pharmacognosy, Faculty of Pharmacy, Cairo University, Kasr El-Aini Street, Cairo 11562, Egypt

2 Department of Pharmacognosy, Faculty of Pharmacy, Egyptian Russian University, Cairo-Suez Road Cairo, Badr City 11829, Egypt

3 Department of Physiology, Faculty of Medicine, Suez Canal University, Ismailia, Egypt

4 Department of Pathology, Faculty of Medicine, Al-Azhar University, Cairo, Egypt

5 Department of Pharmacology and Toxicology, Faculty of Pharmacy, Suez Canal University, Ismailia 41522, Egypt
6 Department of Pharmacology and Toxicology, Faculty of Pharmacy, University of Tabuk, Tabuk, Saudi Arabia

7 Research and Development Functional Food Centre (CIDAF), Bioregión Building, Health Science Technological Park, Avenida del Conocimiento S/N, 18016 Granada, Spain

8 Department of Analytical Chemistry, Faculty of Sciences, University of Granada, Avenida Fuentenueva s/n, 18071 Granada, Spain

9 Department of Pharmacognosy, Faculty of Pharmacy, Al-Azhar University, Cairo, Egypt 